\title{
ESTRATEGIAS EFICACES DE INTERVENCIÓN SOCIOEDUCATIVA CON ADOLESCENTES EN RIESGO SOCIAL ${ }^{1}$
}

\author{
EFFECTIVE STRATEGIES \\ OF SOCIO-EDUCATIONAL INTERVENTION WITH ADOLESCENTS IN \\ SOCIAL RISK SITUATION
}

\section{ESTRATÉGIAS EFICAZES DE INTERVENÇÃO SÓCIO-EDUCATIVA COM ADOLESCENTES EM SITUAÇÃO DE RISCO}

\author{
Miguel Melendro Estefanía, Ángel Luis González Olivares \\ y Ana Eva Rodríguez Bravo \\ Universidad Nacional de Educación a Distancia, España
}

RESUMEN: Si bien la caracterización de la población adolescente y juvenil en riesgo de exclusión social ha sido ampliamente investigada, no lo ha sido tanto la intervención que se realiza con ella. Este artículo hace referencia a una investigación que, realizada entre los años 2011 y 2012, avanza en la descripción de estrategias eficaces de intervención, en su tipificación y estudio, de forma que sirvan como banco de información para una buena práctica profesional, reproducible y útil para mejorar la situación de los adolescentes en riesgo.

Desde los planteamientos de la investigaciónacción, las aportaciones de los profesionales que trabajan directamente con esta población, recogidas a través de cerca de cincuenta cuestionarios y siete grupos de discusión, son una fuente de conocimiento directa y bien documentada, útil para destacar los elementos más relevantes de esta intervención, así como los obstáculos, limi- taciones y prácticas mejorables en este ámbito de trabajo.

Entre otras cuestiones, forman parte de los resultados y la discusión en torno a ellos las propuestas de intervención eficaz sobre las dinámicas familiares conflictivas, la preocupante y cada vez más presente violencia ascendente, el duelo migratorio adolescente y sus efectos de riesgo y marginación, la escasa y frágil participación de los adolescentes en la toma de decisiones sobre su propio proyecto vital y las importantes necesidades formativas del colectivo que trabaja con esta población.

Se perfilan así mismo relevantes elementos estratégicos como base de la intervención con adolescentes en riesgo, entre ellos la flexibilidad para abordar situaciones inciertas, el vínculo, la empatía y la proximidad afectiva como herramientas necesarias en la acción socioeducativa, la contextualización de la intervención en situaciones de conflicto 
y el debate en torno a la resiliencia y sus aportaciones en este ámbito de la Pedagogía Social.

PALABRAS CLAVE: Adolescencia; grupo de alto riesgo; educación social; intervención; investigación acción; educador.

ABSTRACT: Although the characterization of teenagers and young population at risk of social exclusion has been thoroughly investigated, that's not the case of the intervention strategies used. This article refers to a research performed between 2011 and 2012 which advances on the description, categorization and study of effective intervention strategies, so they can be used as an information source for good professional performance, reproducible and useful to improve the situation of teenagers at risk.

From a research-action approach, the contributions from the professionals working with this population, collected from about a hundred tests and seven discussion groups, are a direct and well-documented source of knowledge. This information is useful in order to underline the most relevant elements of this intervention, as well as the obstacles, limitations and practices that can be improved in this field of work.

Part of the results and the discussion about them are, among others, the proposals of effective intervention in conflictive familiar dynamics, the worrying and increasing violence, teenagers migratory grief and its effects of risk and marginalization, the limited and weak participation of teenagers in their own life decision making and the important educational needs of the group of people working with this population.

Furthermore, relevant strategic elements are shaped as the base of the intervention with teenagers at risk situation. Among those elements we find the flexibility to deal with uncertain situations, the link, empathy and affective proximity as necessary tools in socio-educational action, the contextualization of intervention in conflict situations and the debate about resilience and its contributions to the field of Social Pedagogy.

KEYWORDS: Teenager; high risk group; social education; research-action; intervention; educator.
RESUMO: Embora a caracterização de adolescentes e população jovem em risco de exclusão social tem sido exaustivamente investigada, que não é o caso das estratégias de intervenção utilizadas. Este artigo refere-se a uma pesquisa realizada entre 2011 e 2012, que avança sobre a descrição, categorização e estudo de estratégias de intervenção eficazes, de modo que possam ser utilizados como fonte de informação para o bom desempenho profissional, reprodutível e útil para melhorar a situação dos adolescentes em situação de risco.

A partir de uma abordagem de pesquisa-ação, as contribuições dos profissionais que trabalham com esta população, coletados a partir de cerca de uma centena de testes e sete grupos de discussão, são uma fonte direta e bem documentada do conhecimento. Esta informação é útil para sublinhar os aspectos mais relevantes desta intervenção, bem como os obstáculos, limitações e práticas que podem ser melhorados neste campo de trabalho.

Parte dos resultados a discussão sobre eles são, entre outros, as propostas de intervenção eficaz na dinâmica familiar de conflito, a violência preocupante e crescente, adolescentes luto migratório e seus efeitos de risco e da marginalização, a participação limitada e fraca de adolescentes em sua tomada de decisão própria vida e as necessidades educacionais importantes do grupo de pessoas que trabalham com essa população.

Além disso, os elementos estratégicos relevantes são moldados como a base da intervenção com adolescentes em situação de risco. Entre esses elementos, encontramos a flexibilidade para lidar com situações incertas, a ligação, empatia e proximidade afetiva como ferramentas necessárias em ação sócioeducativa, a contextualização de intervenção em situações de conflito o debate sobre resiliência e suas contribuições para o campo da Pedagogia Social.

PALAVRAS-CHAVE: Adolescente; grupo de alto risco; a educação social; a investigação-acção; intervenção; educador.

[ 106 ] MIGUEL MELENDRO, ÁNGEL LUIS GONZÁLEZ OLIVARES, ANA EVA RODRÍGUEZ BRAVO

SIPS - PEDAGOGIA SOCIAL. REVISTA INTERUNIVERSITARIA [1139-1723 (2013) 22, 105-121] TERCERA ÉPOCA 


\section{Introducción}

Debido a sus características y particularidades, la adolescencia es un momento evolutivo que exige una especial atención ante la aparición y consolidación de situaciones de riesgo y de exclusión social $y$, en consecuencia, de vital importancia para el desarrollo, desde el prisma de la intervención socioeducativa, de acciones de prevención y tratamiento de futuras problemáticas.

Algunos estudios recientes que definen las características de los/as adolescentes en riesgo de exclusión social, describen el contexto en el que se desenvuelven e identifican factores e indicadores de riesgo, de protección y/o de resiliencia presentes en su realidad (Lahire, 2007; Rees et al, 2010; Yergeau, Pauzé y Toupin, 2007).

Otros trabajos a tener también muy en cuenta al abordar la temática de los/as adolescentes en riesgo de exclusión social, son los que indagan en las "trayectorias fallidas" de las poblaciones más vulnerables; bien profundizando en el reconocimiento de éstas trayectorias (Fernández del Valle, 1998; García Barriocanal, Imaña y De la Herrán, 2007; Inglés, 2005); bien planteando la necesidad de reestructurar las políticas sociales de atención a estos colectivos (Bendit y Stokes, 2004; Cachón, 2004; Du Bois- Machado y Pohl, 2004; Reymond y López Blasco, 2004).

Así mismo, es preciso no perder de vista aquellas investigaciones que recogen la preocupación actual de las instituciones públicas europeas por el bajo rendimiento escolar de los/as jóvenes, las elevadas tasas de abandono escolar temprano y los condicionamientos que ambos factores ejercen sobre la iniciación al mundo laboral (Casas y Montserrat, 2009; Simon y Owen, 2006), junto a aquellas que ponen de manifiesto la necesidad de profundizar en el apoyo institucional a ésta población a través de "políticas de transición", es decir, de políticas sociales que tengan en cuenta sus especiales características y necesidades (Bendit y Stokes, 2004; Du Bois-Reymond y López Blasco, 2004).

La complejidad del contexto en que crecen los adolescentes en riesgo de exclusión social y la diversidad de los entornos de los que proceden -centros y recursos para menores tutelados de los sistemas de protección, grupos familiares de riesgo, centros para el cumplimiento de medidas judiciales, ...- son elementos determinantes a la hora de entender el concepto de intervención "socioeducativa" e indicadores de la flexibilidad que ésta requiere, tanto para poder acceder de forma adecuada a éstas realidades como para dar continuidad a la acción socioeducativa iniciada, y para estar en condiciones de prepararlos y acompañarlos para su futura y corta trayectoria hacia una vida adulta autónoma y responsable.

Por intervención "socioeducativa", en el marco de esta investigación, hemos entendido una acción social de naturaleza y alcance educativo, en la cual la expresión "socioeducativa" pretende enfatizar la necesidad de una educación que "dé respuesta a un complejo entramado de necesidades sociales de los individuos, restableciendo y ampliando las oportunidades educativas de las personas y de los colectivos sociales en la vida cotidiana" (Caride, 2005, p.57). Además, queremos destacar nuestra comprensión de ésta intervención como praxis, como interacción constante entre acción y reflexión, como práctica compleja, como una "disciplina de acción que se ve empujada a gestionar la incertidumbre, a pactar con el riesgo, a asumir la aleatoriedad inherente a toda acción humana" (Meireiu, 2001, p.109).

En relación a sistemas complejos y abiertos, como el que estamos investigando, Edgar Morin (2005) señala la necesidad de actuar en determinadas condiciones atendiendo a estrategias, más que a programas. Siguiendo esta idea, establecer una secuencia de acciones que deben ser ejecutadas sin variación en un entorno estable, es decir, un programa de acción, es fundamental: Pero cuando existen variaciones importantes y constantes en las condiciones exteriores, ante las cuales es habitual que los programas se bloqueen, la acción se burocratice y se recurra a soluciones estandarizadas, poco prácticas ante fluctuaciones importantes o repetidas, valoramos que es mucho más interesante actuar a través de estrategias. Sin embargo, llegados a este punto cabe preguntarse qué estrategias utilizar y 
cómo hacerlo. Ésta investigación ha avanzado en la descripción de aquellas estrategias que a los profesionales del ámbito socioeducativo les resultan más eficaces en su intervención con adolescentes en riesgo de exclusión social, profundizando en su tipificación y estudio. Pretendemos de esta forma que sirvan como banco de información para una buena práctica profesional, reproducible y útil para mejorar la situación de los adolescentes en riesgo.

Con objeto de avanzar en la caracterización y aplicación del concepto de estrategia al ámbito que nos ocupa, hemos partido de una definición de estrategias eficaces ya utilizada en investigaciones anteriores (Melendro, 2007 y 2010), que tras un nuevo contraste con los profesionales e investigadores implicados en este trabajo quedó conceptualizada de la siguiente manera: procesos de intervención y toma de decisiones a corto y medio plazo -no son meras formulaciones de intenciones- que suponen actuaciones bien definidas, capaces de movilizar sistemas.

Finalmente hemos revisado y analizado, los resultados y aportaciones de los estudios más actuales sobre estrategias de intervención con adolescentes en riesgo de exclusión social. Entre ellos se encuentran investigaciones sobre estrategias relacionadas con políticas integrales (Aguado, 2005; Brullet y Gómez-Granell, 2008; Guasch y Ponce, 2005; Lamarca y Barceló, 2006; Pantoja y Añaños, 2010; Parazelli, 2000; Rivard, 2004; Roldán y Moñivas, 2001), estrategias desde la prevención, la escuela y la educación familiar (Biehal, 2005; Catalano, Haggerty, Harachi y Abbott, 1998, 2005, 2009, 2010; Hicks y Stein, 2010; Iglesias, 2008; Lila, Buelga y Musitu, 2006; Rudduck y Flutter, 2007), estrategias desde el ámbito de la protección y la atención a población en riesgo grave de exclusión social (Balsells, 2006; García Barriocanal, Imaña y De la Herrán, 2007; Harwood, 2009; Minore y Hopkins, 2003; Montserrat y Casas, 2010), estrategias en clave de resiliencia, (Barudy y Dantagnan, 2005; Cirulnik, 2002; Tisseron, 2007) y relacionadas con estrategias para el tránsito a la vida adulta (Fernández del Valle, 1998, 2007, 2009; Goyette et al., 2007; Melendro, 2007 y 2010).

\section{Metodología}

La finalidad de la investigación ha sido la de avanzar en el conocimiento de estrategias eficaces de intervención con adolescentes en riesgo: estrategias contrastadas, validadas y que puedan ser reproducidas con éxito en diferentes contextos de actuación. De acuerdo con esta finalidad, la investigación persigue dos objetivos centrales:

1. Optimizar, desde procesos de investigación-acción, la intervención socioeducativa que se desarrolla con adolescentes y jóvenes en riesgo de exclusión social.

2. Aportar información relevante sobre estrategias eficaces y contrastadas en este ámbito de intervención, favoreciendo su incorporación al trabajo cotidiano de los equipos socioeducativos.

La presencia de múltiples elementos en la atención educativa a los adolescentes en riesgo de exclusión social y la complejidad del diseño mismo de la intervención socioeducativa, nos llevaron a identificar los planteamientos de la Investigación-Acción (IA) y de la Grounded Theory (TF) como los enfoques metodológicos más adecuados al ámbito y las características fundamentales del estudio.

Desde el enfoque de la IA, han sido de utilidad para la investigación algunos planteamientos como: su interés por tratar problemas concretos en el contexto en el que se producen e implicando -de forma colaborativa- a investigadores, profesionales y otros grupos de interés (Cohen y Manion, 1994); su intención, en contraposición a la investigación tradicional, de estudiar una situación social con el fin de mejorar la calidad de la acción dentro de la misma (Elliot, 1993); y la priorización que realiza de las estrategias de actuación como componente central de los programas de intervención, así como al interés por concretar aquellas acciones socioeducativas que pueden resultar más eficaces en los aspectos micro y mesosistémicos (Belsky 1993; Bronfrenbrenner, 1979).

[ 108 ] MIGUEL MELENDRO, ÁNGEL LUIS GONZÁLEZ OLIVARES, ANA EVA RODRÍGUEZ BRAVO 
La TF (Teoría Fundamentada o Grounded Theory) ha sido el soporte metodológico fundamental para el diseño, elaboración y desarrollo de las diferentes partes del proceso de investigación. Se trata de una metodología que ha sido empleada en múltiples investigaciones de diferentes disciplinas, desde la medicina a la antropología, la psicología, la sociología o la propia educación. Así, en nuestro trabajo las fases de investigación completadas han seguido las recomendaciones de Glasser y Strauss (1965) y sus adaptaciones por Andreu, J., García-Nieto, A. y Pérez Corbacho, A.M. (2007) y Trinidad, A., Carrero, V. y Soriano, R.M. (2001), en cuanto al muestreo teórico, la codificación selectiva y teórica de los datos -hasta su saturación- y el proceso de categorización, que han conducido a los resultados que a se reflejan en este trabajo.

El sujeto central del estudio han sido los profesionales que trabajan con los y las adolescentes en riesgo de exclusión social atendidos desde los servicios sociales, con edades comprendidas entre los 12 y los 16 años

En el diseño de las técnicas e instrumentos y en el tratamiento de la información se ha utilizado una metodología mixta, combinando el enfoque cuantitativo, que ha permitido la realización de una buena caracterización del contexto y el contraste de la eficacia de las actuaciones, con el enfoque cualitativo, que ha facilitado la profundización y triangulación de los resultados.

Como técnica de investigación para la recogida de datos de carácter cuantitativo se ha utilizado la encuesta, que se ha aplicado mediante un cuestionario semiestructurado con preguntas cerradas y abiertas. En total en la Comunidad de Madrid, ámbito geográfico al que hace referencia este artículo, se han recogido un total de cuarenta y tres cuestionarios. El análisis de la información aportada por estos cuestionarios se ha realizado básicamente a partir del uso de frecuencias, distribuciones y relaciones entre las variables relevantes para el análisis, utilizando el programa SPSS (versión 19).

Para la recogida de datos de carácter cualitativo, por su parte, se ha utilizado como técnica de investigación el grupo de discusión. Se diseñaron un conjunto de ocho sesiones de discusión en las que participaron un total de quince profesionales. El análisis del contenido de los discursos recogidos en estos grupos ha sido analizado utilizando como herramienta el programa Atlas-ti, versión 6. 2., siguiendo el enfoque metodológico de la Gronded Theory.

\section{Caracterización del escenario de intervención. Resultados del análisis cuantitativo}

El conocimiento de la realidad sobre la que actúan los profesionales es una fuente de información enormemente relevante en investigación; en ella se ven reflejadas las expectativas y motivaciones que surgen en su quehacer cotidiano, conformando un marco enriquecedor sobre las situaciones que requieren un mayor detenimiento en su estudio y valoración. A continuación analizamos una serie de cuestiones que nos parecen relevantes para contextualizar la intervención socioeducativa de los equipos de profesionales que trabajan con adolescentes en riesgo, tras recoger su opinión a través de un cuestionario semiestructurado.

Este grupo de profesionales representan a entidades sociales y a instituciones públicas que trabajan directamente con adolescentes en riesgo de exclusión social. La muestra seleccionada está formada por 43 personas, de las cuales la mayoría son mujeres (65,1\%) y el resto hombres $(34,9 \%)$. Las entidades a las que representan son en su mayoría (80\%) entidades especializadas y con gran experiencia en este ámbito de intervención; el resto son profesionales de instituciones públicas, fundamentalmente de los servicios socioeducativos que atienden a esta población. La formación de estos profesionales destaca por su preparación universitaria (95,3\%) y su formación especializada. En su gran mayoría se trata de profesionales formados inicialmente en carreras como Psicología (23,3\%), Educación Social (16,3\%), Pedagogía (11,6\%) y Trabajo Social (11,6\%).

En la investigación que nos ocupa, el cuestionario semiestructurado utilizado estaba constituido por 30 ítems, que interrogaban a los profesionales sobre las características de los adolescentes con 
los que trabajan, de sus familias y sobre aspectos concretos de la intervención socioeducativa desarrollada con ellos. Atendiendo a estadísticos descriptivos, indicamos a continuación las valoraciones con puntuaciones más significativas en los diferentes ítems del cuestionario. Para ello se ha tenido en cuenta el grado de acuerdo-desacuerdo de los profesionales, sustantivado en una calificación 0-10, donde 0 es el valor más bajo de acuerdo y 10 el más alto (Tabla 1).

Así, los dos ítems que reciben la valoración más alta por parte de los profesionales son los que se refieren al elevado nivel de fracaso escolar de los adolescentes (ítem $20, x=8,32$, ds 1,254) y a la repercusión negativa del fracaso escolar en sus trayectorias (ítem 21, $x=8,34$, ds. 1,47). Los profesionales están seriamente preocupados por la trayectoria de fracaso y abandono escolar de los adolescentes, y consideran que este condicionante afectará gravemente a su futuro.

Con una media muy elevada también encontramos las respuestas al ítem 10 ( $x=8,24$, ds. 1,35), que hacer referencia al diseño, por parte de los profesionales y en colaboración con los adolescentes, de itinerarios personales flexibles, adaptados a las necesidades y posibilidades de cada uno de ellos/a. En este sentido se plantea cómo los profesionales que trabajan con este colectivo tienen una visión estratégica y participativa de su propia actuación, ofreciendo las orientaciones más adecuadas, modificándolas de acuerdo con los adolescentes para alcanzar un mejor desarrollo personal y social. La especial sensibilidad de los profesionales y su profesionalidad en sí se refleja en esta respuesta, donde la intervención se hace a la medida del adolecente y desde su protagonismo.

En sentido inverso, las puntuaciones con menor valor corresponden a los ítems 18 y 19. En el primero de ellos los profesionales responden de manera general que cuando se encuentran con problemas, los adolescentes no cuentan con una red social de apoyo diferenciada de los apoyos institucionales $(x=4,83)$. Las respuestas con una moda de 6 y una $d s=1,773$ describen una ligera divergencia en las opiniones de los profesionales, aunque parece claro el acuerdo general de que no existen redes de apoyo adecuadas para este colectivo desde el ámbito social. Algo similar ocurre con las respuestas al ítem 19, sobre el interés -percibido por los profesionales- de los adolescentes para estudiar y mejorar su formación. En sus respuestas, los profesionales han tenido elecciones próximas al "muy en descuerdo" ( $x=4,76$, moda $=4)$, aunque también ha existido una cierta disparidad en las opiniones ( $d s=1,655)$, en cualquier caso poco significativa.

Entre las respuestas que se encuentran en el punto medio en cuanto a la valoración de los profesionales ( 5 puntos sobre 10), es decir, ítems en los que los profesionales no se decantan ni por el acuerdo ni por el desacuerdo, podemos destacar el ítem $22(x=5,10$, ds 1,446), que pregunta sobre si los adolescentes muestran un comportamiento social responsable al inicio de la intervención socioeducativa, y el ítem 13, que plantea que la formación de los profesionales que atienden a esta población se lleva a cabo de forma frecuente y sistemática. En este último los profesionales han tendido a una respuesta media $(x=5,20)$, con una moda de 7 , algo destacable dada la desviación típica correspondiente de $d s=1,978$, una de las más altas del estudio.

Tabla 1. Estadísticos descriptivos del cuestionario a profesionales

\begin{tabular}{lcccc}
\hline Ítems - Variables & Mín. & Máx. & Media & S.d. \\
\hline En relación a las/los profesionales que trabajáis directamente con adolescentes & & & \\
\hline 9. Tenéis un excelente nivel de conocimiento de: & & & & \\
\hline 9.a Entorno Social & 3 & 9 & 6,93 & 1,506 \\
\hline 9.b Entorno Familiar & 5 & 10 & 7,76 & 1,261 \\
\hline 9. c Entorno Escolar & 6 & 10 & 8,07 & 1,191 \\
\hline
\end{tabular}

[ 110 ] MIGUEL MELENDRO, ÁNGEL LUIS GONZÁLEZ OliVARES, ANA EVA ROdRíGUEZ BRAVO SIPS - PEDAGOGIA SOCIAL. REVISTA INTERUNIVERSITARIA [1139-1723 (2013) 22, 105-121] TERCERA ÉPOCA 


\begin{tabular}{lllll}
\hline \multicolumn{1}{c}{ 9.d Entorno Institucional } & 3 & 10 & 7,46 & 1,645 \\
\hline \multicolumn{1}{c}{ 9.e Itinerario Laboral } & 6 & 10 & 7,98 &, 935 \\
\hline 10. Diseñáis y desarrolláis itinerarios personales flexibles & 4 & 10 & 8,24 & 1,356 \\
\hline 11. Trabajáis de forma coordinada e interdisciplinar. & 2 & 10 & 7,83 & 1,745 \\
\hline 12. Disponéis de recursos, estrategias para abordar situaciones imprevistas. & 3 & 9 & 7,10 & 1,499 \\
\hline $\begin{array}{l}\text { 13. Forma frecuente y sistemática de formación de profesionales } \\
\text { que atienden a esta población. }\end{array}$ & 1 & 9 & 5,20 & 1,978 \\
\hline
\end{tabular}

\section{En relación a la intervención socioeducativa}

14. La intervención socioeducativa es eficaz porque favorece la inclusión social de los adolescentes.

\begin{tabular}{llllll}
\hline 15. La intervención socioeducativa hace disminuir el absentismo escolar. & 3 & 10 & 7,46 & 1,518 \\
\hline 16. La intervención socioeducativa hace disminuir el fracaso escolar. & 3 & 10 & 6,78 & 1,651
\end{tabular}

17. Están claramente establecidos los criterios de evaluación de la intervención socioeducativa.

\begin{tabular}{lllll}
\hline 17.a Evaluación Diagnóstica & 3 & 10 & 6,39 & 1,686 \\
\hline 17.b Evaluación en el Proceso & 3 & 10 & 6,61 & 1,563 \\
\hline 17.c Evaluación Resultados & 3 & 10 & 6,73 & 1,633
\end{tabular}

En relación a las/los adolescentes con los que trabajáis

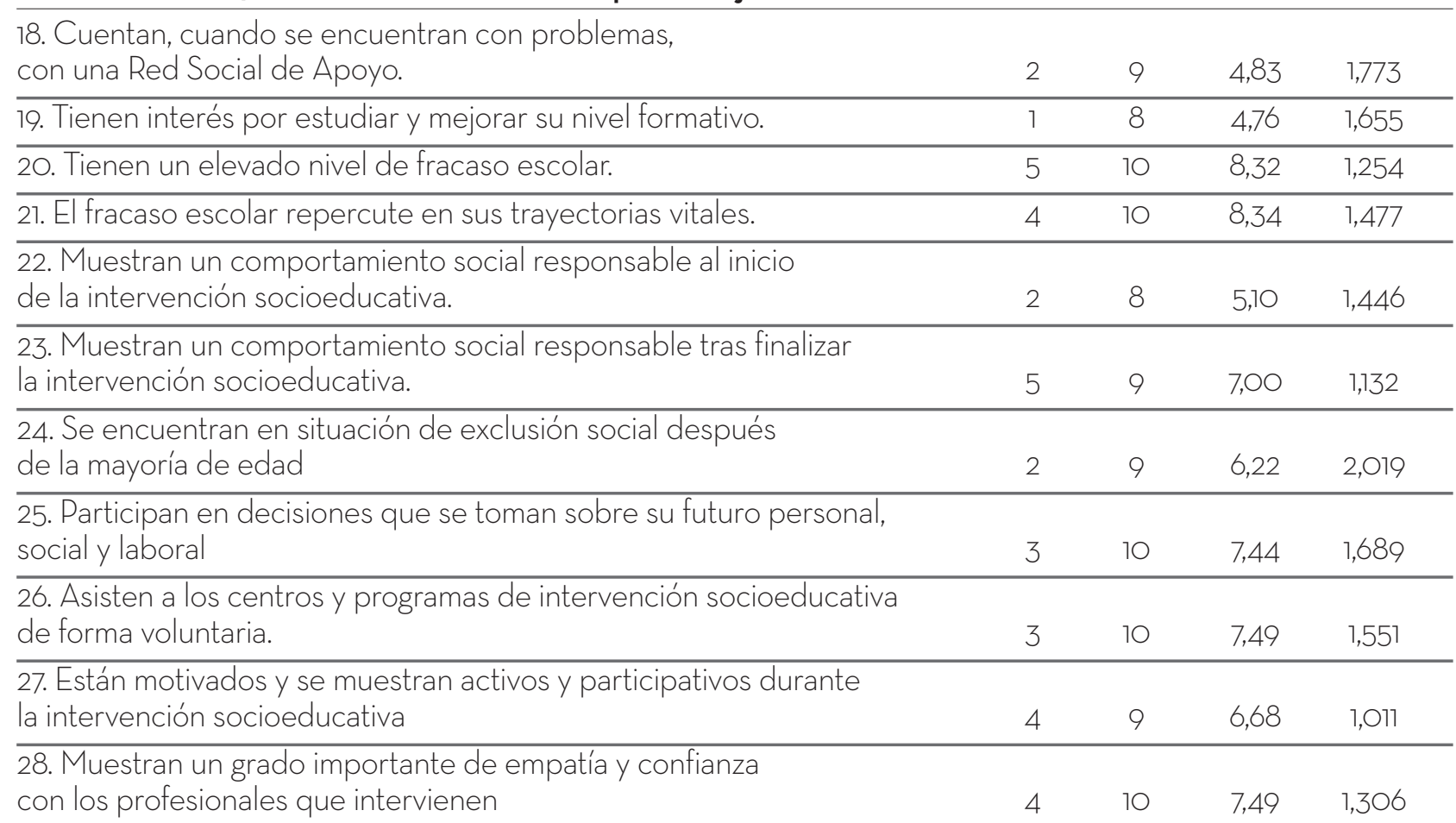

\section{En relación a las familias de las/losadolescentes con los que trabajáis}

29. Se desarrollan intervenciones socioeducativas adecuadas

$\begin{array}{lllll}\text { con los grupos familiares de los adolescentes. } & 3 & 9 & 6,17 & 1,657\end{array}$

\begin{tabular}{lllll}
\hline 30. Las familias colaboran y participan en las actuaciones planteadas. & 2 & 8 & 5,34 & 1,591 \\
\hline
\end{tabular}

Fuente: elaboración propia 
Unas variables muestran, por otra parte, el mayor índice de variabilidad en las respuestas, es decir, suponen aspectos en los que los profesionales no se ponen de acuerdo, y para los que ofrecen valoraciones muy diversas. La primera de ellas hace referencia a la pregunta sobre si prevén que los adolescentes se encontrarán en situación de exclusión social después de la mayoría de edad (ítem 24, $x=6,22, d s=2,019)$. Aunque la media se declina hacia el grado de acuerdo, más detalladamente los valores oscilan entre las puntuaciones de 2 y 9 , justificando su variabilidad por el valor de la desviación típica. En este aspecto los profesionales pronostican, en su mayoría, que los adolescentes en riesgo se encontrarán en situación de exclusión social cuando lleguen a la edad adulta.

Por el contario, los ítems con una menor fluctuación en las puntuaciones, corroborado por una variabilidad menor son el ge y el 27. El primero de ellos hace referencia a que los profesionales que trabajan directamente con adolescentes tienen un excelente nivel de conocimiento de su itinerario y perspectivas laborales ( $9 \mathrm{e}, \mathrm{x}=7,8$ ), con una oscilación de las puntuaciones entre el 6 y el 10, siendo la ds $=0,935$ y la moda de 8 . Es decir, que los profesionales reconocen tener un conocimiento de qué hacer con este colectivo en el ámbito laboral y, además, lo tienen en consideración una amplia mayoría de ellos.

Muy próximo a estas puntuaciones están los resultados del ítem 27, que plantea cómo en la relación de los profesionales con los/las adolescentes con los que trabajan, estos/as están motivados y se muestran activos y participativos durante la intervención socioeducativa que se desarrolla con ellos. Aunque los profesionales han tenido una pequeña variabilidad en las respuestas (puntuación mínima 4 y máxima 9, ds= 1,011), en general la opinión de los técnicos ha sido positiva, describiendo que los sujetos son participativos y muestran interés en la intervención. La motivación y participación tiene una puntuación media de 6,68 y la moda es de 7 .

Analizando de manera general y realizando una clasificación de los valores de la tabla, encontramos una serie de aspectos que los profesionales valoran de forma más positiva (puntuaciones superiores o iguales a 7), otros con los que se encuentran moderadamente satisfechos (puntuaciones iguales o superiores a 6), los que podemos calificar de mejorables (puntuaciones entorno al 5) y manifiestamente mejorables (puntuaciones menores de 5). Comentamos brevemente las variables incluidas en cada unos estos apartados.

Aspectos valorados más positivamente: el conocimiento del entorno familiar, escolar e institucional (ítems $9 b$, 9 c y $9 d$ ) de los adolescentes, junto con la comprensión del itinerario y perspectivas laborales (9e) de los mismos, son ámbitos sobre los que los profesionales afirman tener una información adecuada. De igual modo, el trabajo que se realiza en relación al diseño de itinerarios personales adaptados (10), a las características y circunstancias de los jóvenes, la coordinación interdisciplinar (11), y la disposición de recursos y estrategias para atender a situaciones imprevistas (12) son valorados con como muy adecuados. Además en relación a la intervención socioeducativa, afirman que si esta es eficaz, favorece de forma significativa la inclusión de los adolescentes (14) y disminuye el absentismo escolar (15). Y en relación a los adolescentes con los que se trabaja, se describe que tienen un elevado nivel de fracaso escolar (20), hecho que repercute en su trayectoria vital (21). Aunque también muestran un comportamiento social responsable después de las intervenciones (23) y no les resta autonomía, ya que los adolescentes participan activamente en las decisiones sobre su futuro (25), acudiendo a los centros y programas de intervención socioeducativa de forma voluntaria (26) y mostrando un grado importante de empatía y confianza hacia los profesionales que intervienen socioeducativamente con ellos (28).

Aspectos moderadamente satisfactorios: los profesionales consideran tener un conocimiento adecuado en relación al entorno social de los adolescentes (9a). Por otro lado, en relación a la intervención socioeducativa reflexionan moderadamente que ésta hace disminuir el fracaso escolar (16); de

[ 112 ] MIGUEL MELENDRO, ÁNGEL LUIS GONZÁLEZ OLIVARES, ANA EVA RODRÍGUEZ BRAVO SIPS - PEDAGOGIA SOCIAL. REVISTA INTERUNIVERSITARIA [1139-1723 (2013) 22, 105-121] TERCERA ÉPOCA 
igual modo en el aspecto de la evaluación diagnóstica opinan que están establecidos los criterios a lo largo de todo el proceso de intervención (17a, b y c). En relación a los adolescentes con los que trabajan, pronostican que se encontrarán en situación de exclusión social después de la mayoría de edad (24). Señalan así mismo que los adolescentes están motivados y son participativos durante la intervención (27) y en relación a sus familias, consideran que se desarrollan intervenciones socioeducativas adecuadas con ellas (29).

Aspectos mejorables o que condicionan la intervención: los profesionales describen un conjunto de respuestas próximas al término medio de acuerdo y desacuerdo, entre ellas la formación que reciben los adolescentes en riesgo (13), para unos adecuada y para otros no tanto, pero que requiere necesariamente de una mejora. También la participación de los adolescentes en el inicio de las intervenciones (22) exige una revisión, así como su sentimiento de soledad por la falta de colaboración de sus grupos familiares en las actuaciones planteadas.

Aspectos manifiestamente mejorables o que limitan seriamente la intervención: los profesionales muestran un grado de desacuerdo importante en relación a la suficiencia de las redes sociales de apoyo a los adolescentes en riesgo (18), con independencia de los recursos que ponen a su disposición las instituciones y administraciones que les atienden. Además reflejan que este colectivo no tiene interés por estudiar ni mejorar su nivel formativo (19), motivo que obliga a plantearse que es urgente modificar tanto la percepción de la sociedad hacia ellos como la percepción de ellos hacia la sociedad.

Todos estos argumentos nos hacen tener un conocimiento más cercano y real del contexto en que se produce la intervención socioeducativa con los adolecentes en riesgo de exclusión social, y especialmente la perspectiva de los técnicos y profesionales que trabajan con este colectivo, nos ofrece una información valiosa sobre las prioridades y sugerencias para mejorar la intervención con este colectivo.

\section{Una intervención compleja. Resultados del análisis cualitativo}

Junto al anterior análisis cuantitativo, se ha realizado un análisis cualitativo de la información obtenida en diferentes grupos de discusión de profesionales expertos en la intervención socioeducativa con adolescentes en riesgo. En la experiencia han participado quince profesionales -once educadores/as y cuatro psicólogos/as- pertenecientes a los equipos socioeducativos que intervienen directamente con adolescentes en riesgo, organizados en dos grupos de discusión. La formación inicial de estos profesionales está mayoritariamente relacionada con la psicología (47\%) y la educación social y el trabajo social (33\%); nueve eran mujeres y seis hombres. Se desarrollaron con ellos/ellas ocho sesiones -cuatro con cada grupo- que abordaron diferentes dimensiones de la intervención socioeducativa con adolescentes en riesgo de exclusión social. Todos ellos cumplimentaron, así mismo, el cuestionario a profesionales que se ha descrito en el apartado anterior.

La transcripción de cada uno de los grupos de discusión constituye un documento primario que, una vez codificado, ha facilitado la elaboración de categorías centrales, la emergencia de procesos sociales básicos y la fundamentación de hipótesis teóricas comprensivas de los fenómenos abordados.

Una primera tarea con los grupos de discusión consistió en concretar el concepto de "estrategia eficaz", a partir de una definición previa, procedente de investigaciones anteriores (Melendro, 2007 y 2011), ya que era un elemento central del debate que se iba a establecer posteriormente, así como de la propia investigación.

Otro concepto clave tratado a lo largo de las sesiones fue el de intervención socioeducativa, que es identificada mayoritariamente con un enfoque ecosocial y procesual de la acción socioeducativa, 
muy relacionado con las actuaciones en y sobre los diferentes entornos en que interacciona el/la adolescente. En algunos casos, sin embargo, los profesionales la interpretan de forma ambigua y dispersa, como proceso amplio, genérico, difícil de precisar, e incluso acudiendo a "lo que no es" para poder situarla teóricamente.

A partir de este trabajo previo de conceptualización, se fueron debatiendo, en las diferentes sesiones, una serie de cuestiones relacionadas con las dimensiones principales de la investigación: adolescencias, entornos de riesgo y protección, profesionales intervinientes, sentido de la intervención y estrategias socioeducativas con adolescentes y su entorno. Estas tres últimas dimensiones son las que ocuparon especialmente los tiempos de los grupos de discusión; a ellas se refieren los resultados resumidos a continuación y que han sido sintetizados en la siguiente red conceptual (network) (Gráfico 1).

Gráfico 1. Red conceptual (network) de la investigación

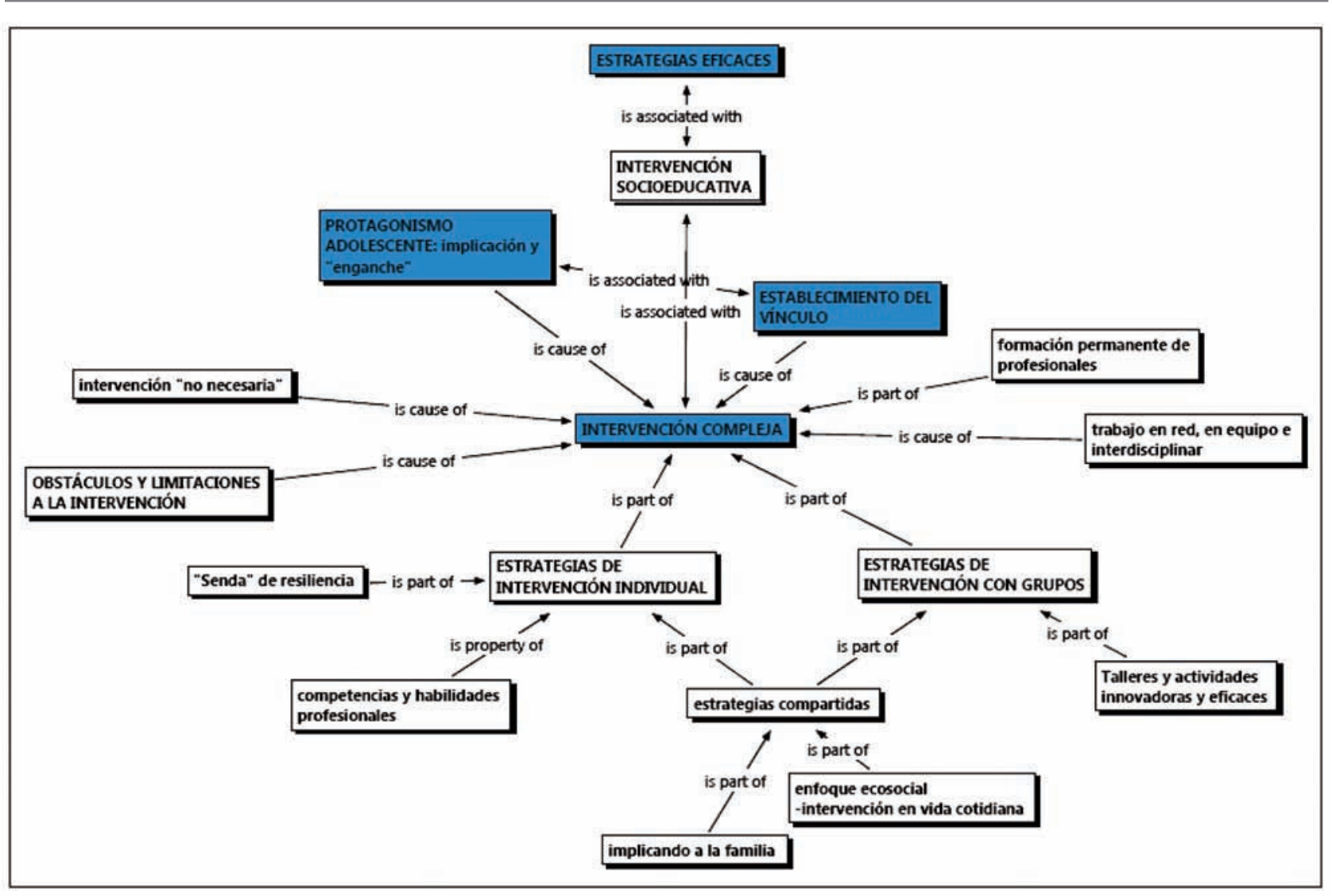

Fuente: Eurostat

En la red conceptual elaborada se destacan tres categorías centrales, fuertemente relacionadas entre sí: el protagonismo adolescente, el establecimiento del vínculo y la complejidad de la intervención.

La primera de estas categorías, se refiere al papel central de los propios adolescentes, la necesidad de contar con su implicación y protagonismo. Sin este elemento, sin el "enganche" de los chavales, el resto de elementos de la intervención pierden en gran medida su eficacia.

La segunda categoría central alude al establecimiento del vínculo entre el profesional y el /la adolescente, que es reconocido como una consecuencia necesaria y a la vez una tarea compleja de la intervención socioeducativa. Más compleja si cabe teniendo en cuenta, como manifiestan de forma repetida los profesionales, que los adolescentes no sienten inicialmente que esa intervención sea necesaria. 
E5: Hay chicos que no necesitan nada de nosotros, o sea ellos no demandan nada y muchas veces pues se las tienes que sacar un poco ahí dependiendo de lo que ves, y otros incluso no quieren nada de nosotros. (M_GDP2_S3) ${ }^{2}$

Y también qué no están habituados a participar activamente o tomar decisiones en este tipo de contexto.

E4: El trabajador social les dice lo que tiene que hacer, el educador familiar, nosotros, en el instituto, o sea aquí opina todo el mundo al final menos ellos y se vuelven como súper automáticos y en el momento en que tú les dices 'bueno y tú qué piensas' se quedan como... como bloqueados, les cuesta un montón incluso en los talleres de creatividad 'y qué hago, tú dime lo que tengo que hacer, es mucho más fácil'. Entonces favorecer esa participación es complicado. (M_GDP1_S3)

La tercera categoría central, destacada en esta parte de la investigación, tiene que ver con la complejidad de la intervención. Como se muestra en la red conceptual (Gráfico 1), esta categoría hace referencia tanto a lo señalado anteriormente, como a otras categorías relevantes que describiremos a continuación: la flexibilidad en el planteamiento estratégico; las modalidades básicas de intervención -individual y con grupos-; el trabajo en red y la formación permanente de los profesionales.

Uno de los elementos más destacados, cuantitativa y cualitativamente, en las aportaciones de los grupos de discusión se refiere a la necesidad de flexibilizar los planteamientos estratégicos con la población adolescente. Este principio de intervención, que hemos resumido en la categoría "estrategias flexibles", implica actuaciones que se pliegan a las condiciones socioambientales tanto en los tiempos como en los lugares, las características de las personas, de los recursos o de las redes de intervención.

Junto a este planteamiento flexible, dos modalidades de intervención que se combinan e interactúan tanto temporal como espacialmente, la intervención individual y la intervención con grupos, son características del trabajo socioeducativo con adolescentes y en su desarrollo vienen a mostrar de nuevo lo complejo que resulta todo a la hora de trabajar con esta población.

Hay una serie de estrategias que pueden considerarse comunes a ambas modalidades de intervención -individual y con grupos-, y a ellas nos referiremos en primer lugar.

Una primera línea común está relacionada con algo ya mencionado anteriormente: el enfoque ecosocial de la intervención socioeducativa. Desde él se plantea la importancia de la proximidad del educador y su presencia en el entorno más inmediato, en la vida cotidiana del/de la adolescente para ayudarle a elaborar cuestiones fundamentales en su vida, en ocasiones en momentos o situaciones aparentemente triviales pero que contienen una carga emocional o competencial enorme, y a los que difícilmente se puede acceder de otra manera.

Este acercamiento a la vida cotidiana solo puede hacerse desde el respeto a los ritmos y a las formas de cada adolescente, a su propio "proyecto vital", único e irrepetible, y a la clara conciencia de que los profesionales que "intervienen" lo hacen solo desde una posición muy limitada, en relación a lo que supone el proyecto vital de una persona. Una posición limitada en los tiempos de intervención, en los momentos y espacios de la vida del adolescente que pueden compartirse, en el papel como profesional cuando diferentes instituciones y responsabilidades se implican, se han implicado y seguirán haciéndolo en el tránsito a la vida adulta de estos adolescentes. Como muy bien expresa una de las educadoras de los grupos de discusión:

...vas a desaparecer de su vida, eso es lo peor de todo. Yo creo que ahí, a veces sin querer, somos egoístas. Yo al principio creo que lo era más porque tú entras, estás un año con él de su vida y te piras, y tú te piras y tienes tu casa, tu familia, tu trabajo o no, lo que tengas ¿no?, pero el chaval sigue ahí, sigue ahí con su problema, con su dinámica familiar horrorosa, con su madre con salud mental esquizofrénica, o sea sigue ahí, entonces tú le has ayudado en un momento de su vida, pero creer que tú vas a cambiar eso... (M_GDP1_S3) 
Una segunda línea compartida en la intervención individual y con grupos -en este caso con grupos de madres- tiene que ver con la implicación de la familia. Una implicación que parte de las necesidades de los adolescentes y tiene a estos como referente principal, y que supone una importante labor de eliminación de "etiquetajes", uno de los principales obstáculos en la intervención con esta población.

E6: Entonces una de las cosas que creo que hacemos en este trabajo es romper esquemas... De repente llegan aquí y a lo mejor es un caso de violencia ascendente y tú no es por lo primero que le preguntas. Tú lo primero que le preguntas es 'cte gusta jugar al futbol?' o 'qué aficiones tienes', 'cómo te sientes'. Entonces de repente rompes esquemas y dice "me acaban de "desetiquetar", y ahora ya no soy no sé quien, con este trastorno, con esta conducta, sino que además se están fijando en otras cosas'. Yo creo que es algo que les llama la atención y que hace que enganchen. (M_GDP1_S2)

En cuanto a las estrategias de intervención individual, se destacan en los grupos de discusión varias líneas básicas de trabajo, como se puede apreciar en la red conceptual elaborada (Gráfico 1). Una de ellas tiene que ver con el desarrollo de una serie de competencias concretas de los profesionales que suponen, entre otras cuestiones, la capacidad para analizar conjuntamente con los adolescentes el significado de las cuestiones vitales que les afectan y, antes que esto, de ser capaces de detectarlas y valorarlas en su justa medida. Junto a estas competencias se subrayan, de forma destacada y repetida, una serie de cualidades y habilidades personales necesarias para su implementación, como la escucha activa, la sinceridad y transparencia, las habilidades de comunicación, el manejo del humor y el uso adaptado del lenguaje con los adolescentes, la flexibilidad y capacidad de adaptación, la creatividad, la tolerancia a la frustración, la empatía, la consistencia educativa y, como síntesis de todas ellas, la disposición para abordar el cambio personal que supone este trabajo.

E7: yo con eso lo que veo es que, no sé cómo llamarlo, pero como estar dispuesto tú también a cambiar. Tú estás trabajando con gente que tiene que cambiar, pero a ti se te dan muchas situaciones en las que pues eso, que tienes que ser más paciente, que tienes que tener mayor tolerancia a la frustración. Tú tienes que estar dispuesto también a ver que te equivocas, y que hay cosas tuyas que tienes que fortalecer, o a desarrollar

Las competencias y habilidades desarrolladas por los profesionales están relacionadas directa, aunque no exclusivamente, con sus necesidades formativas, así como con la intensa labor de trabajo en red, en equipo e interdisciplinar que es necesario desarrollar.

En este sentido, se plantean como necesidades de formación permanente de los profesionales las que conciernen al desarrollo de sus competencias y habilidades, así como las que les ofrecen información y facilitan su respuesta a algunas de las limitaciones y obstáculos más destacados en la intervención con el colectivo de adolescentes, como las estrategias de actuación frente a situaciones de violencia ascendente, violencia entre iguales, absentismo y fracaso escolar, diferencias de género o características multiculturales de los grupos familiares atendidos.

Respecto al trabajo en red, en equipo e interdisciplinar, en los grupos se debatió intensamente sobre algunas dificultades importantes, como las que provocan las intervenciones múltiples de compleja coordinación, en ocasiones desde recursos difícilmente compatibles. En este sentido se insistió especialmente en los problemas provocados por la atención fragmentada a las familias, tanto en el tiempo de intervención como en el profesional y la institución desde la que se interviene; algo que da pie a la presencia de numerosos "juegos familiares" con los profesionales y desestabiliza de forma importante la acción socioeducativa. Un ejemplo de ello:

E6. Hay un hándicap importante en el tema de la violencia ascendente y es la coordinación entre los determinados recursos que trabajamos con la familia o con el adolescente, o por lo menos ese es uno de los planos princi-

[ 116 ] MIGUEL MELENDRO, ÁNGEL LUIS GONZÁLEZ OLIVARES, ANA EVA RODRÍGUEZ BRAVO SIPS - PEDAGOGIA SOCIAL. REVISTA INTERUNIVERSITARIA [1139-1723 (2013) 22, 105-121] TERCERA ÉPOCA 
pales que se nos está dando a nosotros en el centro con determinados casos. Desde el recurso que trabaja con la familia se trabaja de una manera, y nosotros trabajamos de otra, y decimos que vamos a trabajar de la misma manera ¿no? Pero luego, a la hora de poner en común lo que estamos trabajando, cuando llegamos a ponerlo en común, vemos que no se están trabajando las mismas cosas... Entonces en algún caso en particular tenemos problemas de competencias; lo mismo tú estás trabajando una cosa y yo estoy trabajando otra completamente diferente y resulta que lo que estamos haciendo es liar a la familia ¿no? (M_GDP2_S2)

La perspectiva general es, en cualquier caso, la de que el trabajo en red es el adecuado.

E3. Mi experiencia con las coordinaciones es buena. Generalmente se trabaja bien, generalmente están por la labor de coordinarse y generalmente hacen su trabajo. Luego hay excepciones, por supuesto, que son nefastas, pero la regla general es que sí que se trabaja coordinadamente. (M_GDP2_S2)

Otra línea destacada en relación a las estrategias individuales de intervención -aunque realmente nunca pueda separarse, más que de forma teórica, este tipo de modalidades de intervención y sus características- tiene que ver con la que hemos denominado, junto a los participantes de la investigación, "la senda de la resiliencia". Una senda marcada por diferentes subcategorías, entre las que destacar las acciones socioeducativas orientadas a:

- Resaltar los valores de los y las "adolescentes resilientes"

- Promover el esfuerzo y la resistencia a la frustración

- Ayudar a construir imágenes positivas, alternativas a las de exclusión y riesgo dominantes

- Potenciar modelos significativos de identificación, haciendo explícita al adolescente la resiliencia materna, paterna y de otros miembros del grupo familiar y/o de iguales, cuando estas existan.

- Superar sus dificultades de proyección en el futuro

Finalmente, en cuanto a la intervención con grupos de adolescentes, los profesionales dedicaron amplios espacios de tiempo a comentar las actividades y talleres que realizan con ellos. Entre los numerosos talleres mencionados, fueron señalados a la vez como innovadores y eficaces los relativos a internet y las redes sociales (Twenty, Facebook,...), "liantes y líos (sobre relaciones de género en grupo), "mamás de hoy" (con madres adolescentes), un activo taller de cocina que es especialmente valorado o el que los educadores denominan"frikitaller":

E7: sí, sí, he hecho un taller, era un taller curioso. Pues de, le llamamos el frikitaller.. Formalmente no se conoce así, pero la realidad es la que hay... (risas) ...entonces, nada, lo llamamos taller de modelismo y miniaturas y los pongo a pintar figuritas de warhemmer. Lo que pasa es que eso enseguida es muy resultón ¿̇abes?, les sube la autoestima. Son chavales muy retraídos y eso les ayuda un poco a relacionarse, y están en un ambiente social relajados.

E2: con un grupo de chavales más frikis ¿no? que pues que no tienen mucha red social, están muy metidos en casa con combi, ordenador y movidas... la verdad es el típico taller que va enfocado a un público mucho más pequeño, pero yo creo que se trabajan cosas muy potentes... por ejemplo eso sí que creo que entra dentro de esa parte, y yo diría creatividad la nuestra porque tú no sabes la creatividad que tenemos que tener para una actividad, otra actividad, otra actividad y otra actividad (M_GDP1_S3)

Hasta aquí, las aportaciones más relevantes del análisis cualitativo realizado, que reflejan las preocupaciones y propuestas en el día a día de la intervención socioeducativa con adolescentes, narradas y debatidas por los profesionales con una amplia experiencia en este ámbito de acción socioeducativa. 
Gráfico 2. Estrategias eficaces y repercusiones sobre las resistencias del contexto adolescente

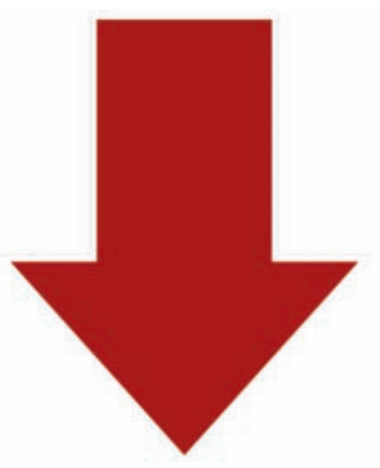

\section{Resistencias del adolescente y su contexto a la intervención}

Vivencia de la intervención como no necesaria.

Obstáculos familiares. Nuevas formas de violencia intrafamiliar.

Desinterés por aprendizajes formales. Fracaso escolar y futura precariedad en el empleo.

Riesgo de exclusión crónica. Grupos de iguales marginales. Bandas. Consumos. Delincuencia.

\section{Estrategias eficaces de intervención socioeducativa \\ Conocimiento amplio y profundo del contexto adolescente que permita flexibilizar la intervención. \\ Actuaciones que facilitan al adolescente la participación y un rol protagonista. \\ Estrategias centradas en el establecimiento y mantenimiento del vínculo. \\ Actuaciones desde elementos básicos de resiliencia. \\ Desarrollo de competencias acordes al marco estratégico.}

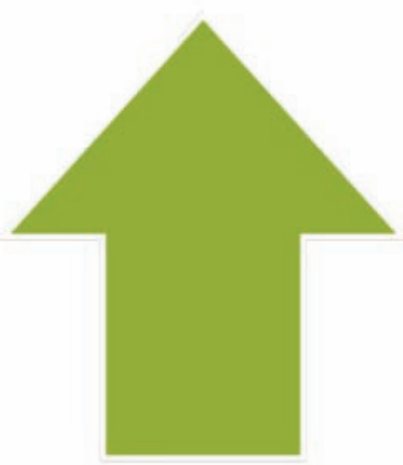

Fuente: elaboración propia

\section{Discusión y conclusiones}

Los resultados obtenidos en la investigación aportan múltiples elementos para avanzar hacia una intervención socioeducativa eficaz. Como se concluye a partir del análisis realizado, estamos refiriéndonos a una intervención sumamente compleja, en la que las claves de su eficacia giran en torno a la puesta en funcionamiento de estrategias flexibles que parten del protagonismo real del adolescente en la toma de decisiones que le son vitales, y que supone como elemento central de la labor de los profesionales el establecimiento del vínculo con estos/as adolescentes.

El enfoque ecosocial de la intervención socioeducativa es otro elemento clave en el trabajo con adolescentes. Como señalábamos anteriormente, desde este enfoque se plantea la importancia de la proximidad del educador y su presencia en el entorno más inmediato, en la vida cotidiana del/de la adolescente para ayudarle a elaborar cuestiones fundamentales en su vida, en ocasiones en momentos o situaciones aparentemente triviales pero que contienen una carga emocional o competencial enorme (Lahire, 2007)

Junto a este enfoque global, aportación diferencial de nuestra investigación, encontramos múltiples elementos que se relacionan parcialmente con investigaciones anteriores en el ámbito que nos ocupa, ya mencionadas en la primera parte de este trabajo.

Así, cabe destacar la importancia que presentan, en relación al establecimiento del vínculo, las aportaciones sobre resiliencia, especialmente las enfocadas a resaltar los valores de los y las "adolescentes resilientes" y de otros modelos de identificación, promover el esfuerzo y la resistencia a la frustración como herramientas para superar las dificultades de proyección en el futuro o ayudar a construir imágenes positivas, alternativas a las de exclusión y riesgo dominantes, en línea con las aportaciones de Tisseron (2007), Cirulnik (2002) o Barudy y Dantagnan (2005). 
Otras investigaciones (García Barriocanal, Imaña y De la Herrán, 2007; Goyette 2004, 2007), señalan también, en sintonía, con nuestras conclusiones, cómo la intervención debe reposar sobre la construcción de una relación fuerte de los equipos socioeducativos con los adolescentes, de forma que el educador se convierta en una persona significativa, clave para ellos y ellas. Más aún cuando, como manifiestan de forma repetida los profesionales, los adolescentes no sienten inicialmente que esa intervención sea necesaria (Rees, et al, 2010)

Mención aparte merece la significativa preocupación de los profesionales por el elevado nivel de fracaso escolar de los adolescentes y la repercusión negativa de este hecho en sus trayectorias vitales. Todo ello unido al escaso interés de los adolescentes por estudiar y mejorar su nivel formativo, supone movilizar una serie de estrategias que, en línea con investigaciones que han tratado este tema con anterioridad, plantean la necesidad de apoyar a los y las adolescentes en la mejora de sus relaciones familiares (Biehal, 2005; Lila, Buelga y Musitu, 2006) y en el establecimiento de relaciones socio-afectivas positivas más allá del entorno familiar y/o con su grupo de iguales (Yergeau, Pauzé y Toupin, 2007) así como la eficacia de una intervención preventiva global, a través de la creación de espacios de apego y de compromiso entre los miembros de la comunidad y la transformación intensiva de los entornos escolares (Catalano, Haggerty, Harachi y Abbott, 1998, 2005, 2009 y 2010).

Esto ha de ir unido a un protagonismo destacado de los adolescentes en la toma de decisiones sobre cuestiones vitales para ellos, tanto individual como grupalmente, sean estas de menor o mayor envergadura (Rudduck y Flutter, 1998, 2001, 2007)

Por último habría que resaltar la necesidad de mejorar las políticas sociales y de la formación de los profesionales, tanto en relación al desarrollo de competencias adecuadas a este ámbito específico de intervención (Bautista-Cerro y Melendro, 2012), como destacando la importancia de saber construir desde la cohabitación de los paradigmas de intervención, a través del trabajo en red e interdisciplinar (Goyette, 2004; Melendro 2007).

Tras esta breve revisión de los aspectos más destacados de nuestra investigación y su relación con otras aportaciones destacadas en el ámbito de la intervención con adolescentes en riesgo, retomamos una de las referencias metodológicas básicas del estudio, la Investigación-Acción (IA). La implicación de profesionales, investigadores, responsables institucionales en esta investigación hace especialmente importante insistir en la necesidad de reflexionar conjuntamente y diseñar acciones formativas y de planificación que incorporen estas propuestas a la intervención socioeducativa que se desarrolla día a día, en torno a la vida cotidiana de los adolescentes. La realimentación a partir de todo ello y la construcción de una atención de calidad a este colectivo, son el objetivo fundamental de la tarea desarrollada, y la base para futuros estudios en este ámbito.

\section{Referencias bibliográficas}

Aguado, P. (2005). Programa de prevención y control del absentismo escolar en el ayuntamiento de Madrid. INDIVISA. Boletín De Estudios e Investigación, 6, pp. 249-257.

Andreu, J., García-Nieto, A. y Pérez Corbacho, A. M. (2007). Evolución de la Teoría Fundamentada como técnica de análisis cualitativo. Madrid: CIS (Centro de Investigaciones Sociológicas).

Balsells, M. Á. (2006). Québec y Cataluña: Redes y profesionales para la acción socioeducativa con familias, infancia y adolescencia en situación de riesgo social. Revista Española De Educación Comparada, 12, pp. 365-387.

Bautista-Cerro, M.J. y Melendro, M. (2011). Competencias para la intervención socioeducativa con jóvenes en dificultad social. Educación $X X_{1}, 14$, pp. 179-200.

Belsky, J. (1993). Etiology of child maltreatment, a developmental-ecological analysis. Psychological Bulletin, 114 (3), pp. 413434.

Bendit, R. y Stokes, D. (2004). Jóvenes en situación de desventaja social: políticas de transición entre la construcción social y las necesidades de una juventud vulnerable. Revista de Estudios de Juventud, 65, pp. 11-29. 
Biehal, N. (2005). Working with adolescents at risk of out of home care: the effectiveness of specialist teams. Children and Youth Services Review, 27 (10), pp. 45-59.

Bronfenbrenner, U. (1979). La ecología del desarrollo humano. Buenos Aires: Paidós.

Brullet, C. y Gómez-Granell, C. (2008). (Coord.). Malestares: infancia, adolescencia y familias. Barcelona: Graó.

Cachón, L. (2004). Las políticas de transición: estrategia de actores y políticas de empleo juvenil en Europa. Madrid: Instituto de la Juventud.

Caride, J. A. (2005). Las fronteras de la Pedagogía Social. Perspectivas científica e histórica. Barcelona: Gedisa.

Casas, F. y Monserrat, C. (2009). Sistema educativo e igualdad de oportunidades entre los jóvenes tutelados: estudios recientes en el Reino Unido. Psicothema, 21 (4), pp. 543-547.

Catalano, R. F., Park, J., Harachi, T. W., Haggerty, K. P., Abbott, R. D., y Hawkins, J. D. (2005). Mediating the effects of poverty, gender, individual characteristics, and external constraints on antisocial behavior: A test of the social development model and implications for developmental life-course theory. En D. P. Farrington. (Ed.). Advances in criminological theory: Vol.14. Integrated developmental and life-course theories of offending (pp. 93-123). New Brunswick, NJ: Transaction.

Cohen, L. y Manion, L. (1994). Métodos de investigación educativa. Madrid: La Muralla.

Du Bois-Reymond, M. y Lopez, A. (2004). Transiciones tipo yo-yo y trayectorias fallidas: hacia las políticas integradas de transición para los jóvenes europeos. Revista de Estudios de Juventud, 65, pp. 11-29.

Elliot, J. (1993). El cambio educativo desde la investigación acción. Madrid: Morata.

Fernández del Valle, J. (1998). Y después... ¿qué? Estudio de casos que fueron acogidos en residencias de protección de menores en el Principado de Asturias. Oviedo: Consejería de Servicios Sociales del principado de Asturias.

García Barriocanal, C., Imaña, A. y De la Herrán, A. (2007). El acogimiento residencial como medida de protección al menor. Madrid: Defensor del menor en la Comunidad de Madrid.

Glasser, B. y Strauss, A. (1967). The Discovery of Grounded Theory: strategies for qualitative research. New York: Aldine de Gruyter.

Goyette, M. y Turcotte, D. (2004) La transition vers la vie adulte des jeunes qui ont vécu un placement: un défi pour les organismes de protection de la jeunesse. Revue Service Social, 51, pp. 29-44.

Goyette, M., Chénier, G., Royer, M.N. y Noel, V. (2007). Le soutien au passage à la vie adulte des jeunes recevant des services des centres jeunesse. Éducation et francophonie. Revue cientifique virtuelle, 35 (1), pp. 95 -119.

Guasch, M. y Ponce, C. (2005). Intervención psicopedagógica: Proyectos y programas de intervención en situaciones de infancia en riesgo social. Universitas Tarraconensis. Revista de Ciències de l'Educació, pp. 215-230.

Harwood, V. (2009). El diagnóstico de los niños y adolescentes problemáticos. Una crítica a los discursos sobre los trastornos de la conducta. Madrid: Morata.

Hicks, L. y Stein, M. (2010). A multi-agency guide for professionals working together on behalf of teenagers. Department for children, schools and families. Recuperado de: https://www.gov.uk/government/uploads/system/uploads/ attachment_ data/file/190250/00247-2010DOM-EN.PDF

Iglesias Galdo, A. (2008). Educar e castigar. Unha historia do presente da xustiza de menores en Galicia. Servizo de Publicacións, Universidade da Coruña.

Inglés, A., et al. (2005). Aprendiendo a volar. Estudio para el análisis de los programas europeos Mentor 15 y Ulises dedicados al proceso de socialización de adolescentes y jóvenes tutelados que han alcanzado la mayoría de edad o están cerca de ella. Murcia: Fundación Diagrama.

Lahire, B. (2007). Infancia y adolescencia: De los tiempos de socialización sometidos a constricciones múltiples. Revista de Antropología Social, 16, pp. 21-38.

Lamarca Iturbe, Í. y Barceló Galdácano, F. (2006). Personas menores en situaciones de especial vulnerabilidad: Retos para una intervención eficaz. Revista de Psicodidáctica, 11(1), pp. 25-36.

Lila, M., Musitu, G. y Buelga, S. (2006). Programa LISIS: las relaciones entre padres e hijos en la adolescencia. Madrid: Pirámide.

Machado Pais, J. y Pohl, A. (2004). Los dilemas del reconocimiento del aprendizaje informal. Revista de Estudios de Juventud, 65, pp. 83-98.

Meirieu, P. (2001). La opción de educar. Ética y pedagogía. Barcelona: Octaedro.

Melendro, M. (2010). El tránsito a la vida adulta de los jóvenes en dificultad social. Madrid: UNED.

Melendro, M. (Dir.) (2007). Estrategias educativas con adolescentes y jóvenes en dificultad social. El tránsito a la vida adulta en una sociedad sostenible. Madrid: UNED, Ayuntamiento de Madrid y Opción 3 Soc. Coop.

[ 120 ] MIGUEL MELENDRO, ÁNGEL LUIS GONZÁLEZ OLIVARES, ANA EVA RODRÍGUEZ BRAVO

SIPS - PEDAGOGIA SOCIAL. REVISTA INTERUNIVERSITARIA [1139-1723 (2013) 22, 105-121] TERCERA ÉPOCA 
Minore, B. y H. Hopkins (2003). Suicide Response Plans: A Comparative Cross-Jurisdictional Analysis. Position paper prepared for the Government of Nunavut Task Force on Mental Health, lqaluit, Nunavut: Centre d'excellence pour les enfants et adolescents ayant des besoins spéciaux et Santé Canada.

Montserrat, C. y Casas, F. (2010) Educación y jóvenes ex-tutelados. Revisión de la literatura científica española. Educación $X X I, 13-2$, pp. 117-138.

Morín, E. (1994). Introducción al pensamiento complejo. Barcelona: Gedisa.

Pantoja, L. y Añaños, F. (2010). Actuaciones socioeducativas con menores vulnerables, en riesgo, relacionados con las drogas. Reflexiones críticas. Pedagogía Social. Revista Interuniversitaria, 17, pp. 109-122.

Parazelli, M. (2000). L'imaginaire familialiste et l'intervention sociale auprès des jeunes de la rue : une piste d'intervention collective à Montréal. Santé mentale au Québec, 25-2, pp. 40-66.

Rees, G., Gorin, S., Jobe, A., Stein, M., Medforth, R. y Goswami, H. (2010). Safeguarding Young People: Responding to young people aged 17 to 17 who are maltreated. The children's society. Recuperado de: http://www.york.ac.uk/inst/ spru/pubs/pdf/safeguarding.pdf

Rivard, J. (2004) Des pratiques autour des jeunes / enfants des rues : une perspective internationale. Revue Nouvelles Pratiques sociales, 17-1, pp. 126-148.

Roldán, E., \& Moñivas, A. (2001). Análisis comparativo de estándares de buenas prácticas para estrategias innovadoras de bienestar social ASIPS. proyecto leonardo da vinci. Cuadernos de Trabajo Social, 14, pp. 371-380.

Rudduck, J. y Flutter, J. (2007). Cómo mejorar tu centro escolar dando la voz al alumnado. Madrid: Morata.

Simon, A. y Owen, C. (2006). Outcomes for children in care: what do we know?. En E. Chase, A. Simon y S. Jackson: In care and after: A positive perpective. London: Routledge.

Trinidad, A., Carrero, V. y Soriano, R.M. (2001). Grounded Theory: la construcción de la teoría a través del análisis interpretacional. Madrid: CIS (Centro de Investigaciones Sociológicas).

Yergueau, E., Pauze, R. et Toupin, J. (2007). L'insertion professionnelle et l'adaptation psychosociale des jeunes adultes ayant reçu des services des centres jeunesse. Revue Intervention, 127, pp. 58-69.

\section{Notas}

${ }^{1}$ Este trabajo se inserta en el marco de la investigación CTINV041/11 sobre Estrategias eficaces de intervención socioeducativa con adolescentes y jóvenes en riesgo de exclusión social, financiada por Opción 3 S.C., Igaxes 3, Fundación Isos y Fundación Trébol, en convenio de colaboración con la UNED (Art. 83 LOU) y con la participación del Ayuntamiento de Madrid, la Universidad de A Coruña, la Universidad de Girona, el Grupo de Investigación G44Edu16 - Contextos de Intervención socioeducativa de la UNED y la Fundació Pare Manel.

th. 2 Referencia de territorio, grupo de discusión y número de sesión.

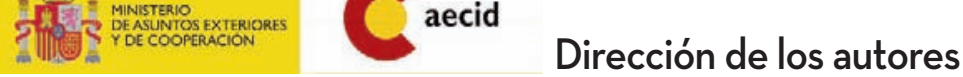

Miguel Melendro Estefanía, Ángel Luis González Olivares y Ana Eva Rodríguez Bravo. Universidad Nacional de Educación a Distancia. Departamento de Teoría de la Educación y Pedagogía Social. Facultad de Educación. C/ Juan del Rosal, 14. 28030 Madrid, (España).

Correo electrónico: mmelendro@edu.uned.es; algonzalez@edu.uned.es; anaeva.rodriguez@edu.uned.es

Fecha de recepción del artículo: 7.2.2013

Fecha de revisión del artículo: 4.3 .2013

Fecha de aceptación final: 25.6.2013

\section{Cómo citar este artículo}

Melendro Estefania, M; González Olivares, A. L. y Rodríguez Bravo, A. E. (2013). Estrategias eficaces de intervención socioeducativa con adolescentes en riesgo social. Pedagogía Social. Revista Interuniversitaria, 22, pp.> 105-121 